\title{
Metodología para la estimación de pérdidas económicas de cultivos de maíz siniestrados por inundaciones usando teledetección
}

\section{Methodology for estimating economic losses of corn crops damaged by floods using remote sensing}

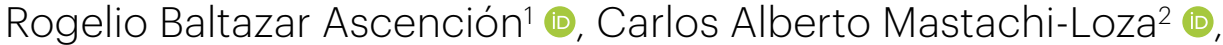 \\ Adalberto Galindo Alcántara ${ }^{3}$ (1), Marivel Hernández Téllez ${ }^{4}$ (1) \\ y Rocio Becerril-Piña ${ }^{5}$ (c)
}

\begin{abstract}
RESUMEN
Se determinaron las zonas inundadas de cultivo de maíz por medio de imágenes de satélite a fin de conocer las pérdidas económicas por inundaciones. Como caso de estudio se analizó una inundación ocurrida en 2016 que afectó 4 municipios del Estado de Tabasco, México. La metodología comprendió la utilización de imágenes de satélite Landsat (L8), cálculo de índices espectrales (agua, vegetación y humedad), identificación de áreas de cultivo de maíz a través de clasificación supervisada y la estimación de las pérdidas económicas. Los resultados muestran que los índices espectrales que mejor lograron identificar las zonas inundadas fueron: MNDWI y NDWI. Para reportar los daños, se estandarizaron los valores en USD (dólares estadounidenses) en precios de 2016 utilizando tipos de cambio ajustados por paridad de poder adquisitivo (PPA). Para el estudio de caso los resultados de daños por hectárea se establecieron entre los 728.57 USD/ha en PPA y los 317.25 USD/ha en PPA los cuales concuerdan con las estimaciones realizadas en otros países para cultivos de maíz.
\end{abstract}

Palabras clave: Inundaciones, Índices espectrales, Cultivos de maíz, Estimaciones económicas.

Instituto Interamericano de Tecnología y Ciencias del Agua (IITCA), Universidad Autónoma del Estado de México. Toluca, México. Correo electrónico: robaas21@hotmail.com

Instituto Interamericano de Tecnología y Ciencias del Agua (IITCA), Universidad Autónoma del Estado de México. Toluca, México. Correo electrónico: camastachil@uaemex.mx

División Académica de Ciencias Biológicas, Universidad Juárez Autónoma de Tabasco. Tabasco, México. Correo electrónico: aga2003a@hotmail.com

Instituto Interamericano de Tecnología y Ciencias del Agua (IITCA), Universidad Autónoma del Estado de México. Toluca, México. Correo electrónico: mhernandezt@uaemex.mx

Red Lerma-Instituto Interamericano de Tecnología y Ciencias del Agua (IITCA), Universidad Autónoma del Estado de México. Toluca, México. Autor para correspondencia. Correo electrónico: rbecerrilp@uaemex.mx 


\begin{abstract}
The flooded areas of corn cultivation were determined by means of satellite images in order to know the economic losses due to floods. As a case study, a flood that occurred in 2016 that affected 4 municipalities in the State of Tabasco, Mexico, was analyzed. The methodology included the use of Landsat satellite images (L8), calculation of spectral indices (water, vegetation and humidity), identification of corn growing areas through supervised classification and the estimation of economic losses. The results show that the spectral indices that best managed to identify the flooded areas were: MNDWI and NDWI. To report damages, values in USD (US dollars) were standardized in 2016 prices using exchange rates adjusted for purchasing power parity (PPP). For the case study, the results of damages per hectare were established between 728.57 USD / ha in PPP and 317.25 USD / ha in PPP, which agree with the estimates made in other countries for corn crops.
\end{abstract}

Keywords: Floods, Spectral indexes, Corn Crop, Economic estimates.

El fenómeno de las inundaciones es uno de los desastres más devastadores del mundo, principalmente por los desbordamientos de ríos de forma repentina, propiciado por fuertes precipitaciones y el desbordamiento de embalses (Sharma et al., 2019), afectando especialmente a la población y algunas actividades económicas, entre estas la agricultura. Los daños por inundaciones se clasifican en daños directos e indirectos. Los primeros son aquellos que se producen por el contacto físico del agua de la inundación con seres humanos y bienes. Los daños indirectos son inducidos por los impactos directos y ocurren en el espacio y tiempo fuera del evento de inundación. En el sector agrícola los daños por inundaciones incluyen pérdidas de productos agrícolas, daño a la infraestructura agrícola, así como afectaciones en la reducción del rendimiento y calidad de la producción. Esto por alteración de la calidad del suelo ya sea por deposición de contaminantes, compactación o erosión del suelo (Merz et al., 2010). A nivel mundial se tienen registros sobre los daños que causan las inundaciones al sector agrícola. EM DAT menciona (EM DAT (Emergency Events Database), 2016), que entre 2003-2013 se registraron 78 desastres, destacando las inundaciones en Pakistán (2010, 2011), Tailandia (2011) y Colombia (2010-2011) que causaron pérdidas de 9.3 billones de USD (Dólares estadounidenses) en el sector agrícola. Si bien es cierto, los impactos de las inundaciones pueden ocurrir en diversas regiones del mundo, los países en desarrollo son particularmente vulnerables a los daños por inundaciones, debido a la limitación de recursos para hacerles frente.

En el caso de México se han tenido una serie de inundaciones, que han provocado daños considerables al sector agrícola. Entre las más importantes por los daños agrícolas ocurridos destacan las ocurridas en 1997 (122,282 ha), 2002 (203,434 ha), 2003 (154,324 ha) y 2007 (93,319 ha) (CENAPRED, 2014). Las pérdidas de maíz fueron un grave impacto en la seguridad alimentaria de los hogares, ya que el $85 \%$ de la producción de maíz es para consumo y es un alimento básico entre la población local (FAO, 2015). Asimismo, entre los daños indirectos destacan la reducción significativa en la producción de carne y leche (FAO, 2015).

A menudo, la evaluación del daño de inundaciones a cultivos se descuida o solo se toma en cuenta mediante el uso de enfoques simples y estimaciones aproximadas (Förster et al., 2008). Los enfoques de estimación varían desde modelos que diferencian solo entre daños a tierras de cultivo y pastizales (Hoes \& Schuurmans, 2006) hasta los que diferencian por tipo de cultivo (Dutta, Herath \& Musiake, 2003; Förster et al., 2008). Para obtener una estimación del daño total esperado, el daño relativo estimado debe estar relacionado con el valor de mercado que podría 
haber sido obtenido por el cultivo cosechado sin inundación. Las estimaciones económicas son importantes, ya que dan un valor aproximado de las pérdidas económicas que se tuvieron, permiten cuantificar lo perdido, así como evaluar posibles escenarios futuros y las pérdidas que se tendrían por un evento como es el caso de una inundación y para dar soluciones al problema, puesto que este va dejando pérdidas de manera regular (Merz \& Thieken, 2009; Merz et al., 2010).

Las aproximaciones para evaluar las inundaciones incluyen: la evaluación directa a través del trabajo de campo (Hernández-Uribe, Barrios-Piña \& Ramírez, 2017; Díaz-Borrego \& Rodríguez-Infante, 2016; Rodríguez Gavíria, 2016; Escuder, Matheu \& Castillo, 2010); entrevistas, medición de tirantes e inventarios (Moguel, Tejeda \& García Pacheco, 2012; Aguilar \& Bedoya, 2006); y el enfoque a distancia, mediante la aplicación de sensores remotos satelitales (Singh, Krishna Kant \& Singh, 2017; Singh, Akansha \& Singh, 2017; Zhou \& Zhang, 2017; Acharya et al., 2016; Fisher, Flood \& Danaher, 2016; Franci et al., 2016; Twele et al., 2016; Jung et al., 2014). La teledetección es una herramienta que en los últimos años ha tenido gran desarrollo, principalmente en aquellas regiones de difícil acceso, como lo podrían ser las zonas inundadas. Los índices espectrales permiten dar seguimiento a la condición de la vegetación y humedad, identificando cambios en la superficie. Entre los índices más utilizados destacan: MNDWI (Modificated Normalized Difference Water Index), NDWI (Normalized Difference Water Index)), diferentes clasificadores (Maximum Likelihood, Kohonen Clustering Network, Support Vector Machines, Isodata), modelos digitales de elevación e interpolaciones IDW (Inverse Distance Weighting).

Las metodologías para evaluar las inundaciones pueden ser complejas en su aplicación debido a la dificultad para la obtención de las variables que utilizan, como la propuesta por Dutta, Herath y Musiake (2003) que es la combinación de un modelo hidrológico distribuido de base física y un modelo de estimación distribuida de pérdidas por inundación. Otra metodología de características similares es la de Baró-Suárez et al. (2012) que utiliza curvas de duración de la inundación-daños, así como modelos hidrológicos e hidráulicos (Vozinaki et al., 2015; Crow, 2014; Jongman et al., 2012; Scawthorn et al., 2006), donde se considera la profundidad de la inundación, velocidad de flujo y simulaciones de inundaciones. Otras metodologías que no incluyen variables de tipo hidrológicas ni simulaciones, se enfocan en datos propios de los cultivos: tipo, rendimiento y área (Balica et al., 2013; Förster et al., 2008). Asimismo, hay metodologías que están en función del riesgo, peligro, vulnerabilidad y resiliencia ante una inundación. (Merz et al., 2010; Merz \& Thieken, 2009).

Finalmente, otras metodologías se apoyan en imágenes de satélite, fotografías aéreas, modelos digitales de elevación e índices espectrales. Son varios los estudios que delimitan la zona de interés de manera general, integran variables hidrológicas, simulaciones, información agrícola y NDVI (Normalized Difference Vegetation Index) (Tapia-Silva et al., 2011; Forte, Strobl \& Pennetta, 2006). La viabilidad o aplicabilidad de los diferentes métodos existentes para estimaciones está en función de la disponibilidad y obtención de la información de entrada, así como el presupuesto disponible que se requiere para aplicarlos.

Debido a lo anterior, el presente trabajo tuvo como objetivo realizar la estimación de las pérdidas económicas por daño a cultivos de maíz afectados por inundación, utilizando la metodología de Förster et al. (2008) mediante el uso de técnicas de teledetección. La metodología se aplicó a 
un caso de estudio al estimar el daño a cultivos de maíz en el ciclo otoño-invierno 2015-2016 en el estado de Tabasco, México.

\section{Material y Métodos}

\section{Caso de estudio: Tabasco, México}

El estado de Tabasco está conformado por 17 municipios y se encuentra localizado en el sureste de México, colinda al norte con el Golfo de México, al este con el estado de Campeche, al oeste con la entidad federativa de Veracruz y al sur con el estado de Chiapas (Figura $N^{\circ} 1$ ). Es una región planicie costera, con una producción agrícola y ganadera importante en la región. En los últimos 25 años Tabasco ha tenido inundaciones significativas como las sucedidas en 1999 (Declaratoria de Desastre Natural), afectando 153,230 personas (DOF, 1999). En 2007 (Declaratoria de Desastre Natural), dejando 32 millones de pesos en pérdidas (DOF, 2007). En 2010 (Declaratoria de Desastre Natural), afectaron a 130,222 personas en 420 comunidades de 13 municipios (Instituto de Protección Civil del Estado de Tabasco, 2016). En 2011 (Declaratoria de Desastre Natural), la entidad tuvo 250,000 damnificados, y 350,000 ha afectadas, esto en 16 de 17 municipios. En 2015 (Declaratoria de Emergencia) se registraron inundaciones en 9 municipios, dejando 71,541 ha de cultivos afectadas (Instituto de Protección Civil del Estado de Tabasco, 2016). Las intensas precipitaciones que se han presentado en la entidad son principalmente debido a la presencia de huracanes como lo fueron Dean (2007), así como frentes fríos y tormentas tropicales, haciendo que los ríos presentes en la zona se desborden. Además, su clima es cálido húmedo en el $95 \%$ de su superficie y cálido subhúmedo en la parte este. La precipitación media estatal es de 2,550 $\mathrm{mm}$ anuales, las lluvias se presentan todo el año, siendo más abundantes en los meses de junio a octubre (INEGI, 2018).

Para este estudio se trabajó con el periodo que comprende la inundación (20 de diciembre de 2015 en adelante), siendo que en los municipios siniestrados existió una precipitación acumulada en tres días seguidos de $74 \mathrm{~mm}$, lo que provocó que cuatro municipios tuvieran afectaciones en cultivos de maíz por las inundaciones. Los municipios tomados en cuenta son Cárdenas, Huimanguillo, Nacajuca y Tacotalpa (Figura $N^{\circ} 1$ ). Se trabajó a nivel municipal debido a que los datos reportados oficialmente por el SIAP (Servicio de Información Agroalimentaria y Pesquera) (SAGARPA, 2018) están estructurados a nivel municipal y en algunos casos los municipios están divididos en CADER (Centros de Apoyo al Desarrollo Rural), por lo cual no es posible delimitar la zona a nivel de subcuenca.

La zona de estudio es importante debido a que siempre ha existido el cultivo de maíz, pero al paso de los años se ha ido relegando la siembra de este cultivo, el cual en cuestiones de seguridad alimentaria para las familias cobra relevancia ya que en México cerca del $45 \%$ del consumo nacional de calorías procede del maíz. En las zonas rurales con bajos recursos, los hombres pueden consumir cerca de $600 \mathrm{~g}$ de maíz, y las mujeres $400 \mathrm{~g}$. (FAO, 2016). Los cultivos cíclicos más importantes son: maíz, arroz, sorgo y frijol (SAGARPA, 2018). Existen programas por parte de SAGARPA que tienen la intención de hacer que el estado de Tabasco se convierta en productor de maíz a nivel nacional, como lo es el Proagro Productivo cuyo objetivo es incorporar 30 mil nuevas ha para maíz en el periodo 2017-2018 y así alcanzar las 106 mil ha dedicadas a este cultivo. 
Figura $\mathrm{N}^{\circ} 1$.

Zona de estudio y municipios siniestrados en Tabasco, México

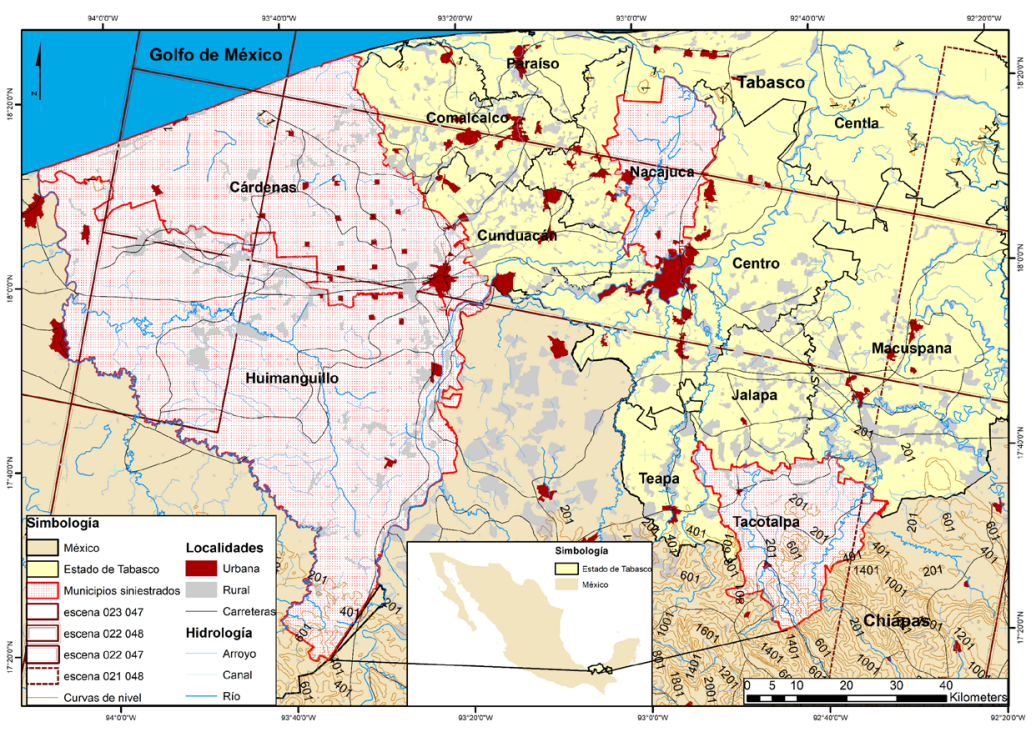

Fuente: Elaboración propia con información cartográfica de INEGI.

En la Figura ํ2 se muestra el periodo que provocó inundaciones y afectó los cultivos de maíz, de manera general se puede establecer del 20 de diciembre del 2015 al 5 de enero del 2016. En la gráfica de barras se observa la precipitación acumulada para cada municipio en el periodo del 20 al 22 de diciembre del 2015, debido a que en estos días las precipitaciones fueron más intensas. Finalmente, se muestran las imágenes Landsat 8 , así como la fecha, columna, fila y el uso que se le dio a cada imagen. Fueron un total de 8 imágenes, distribuidas en 4 escenas las que cubrieron la zona de estudio.

Figura $\mathbf{N}^{\circ} 2$.

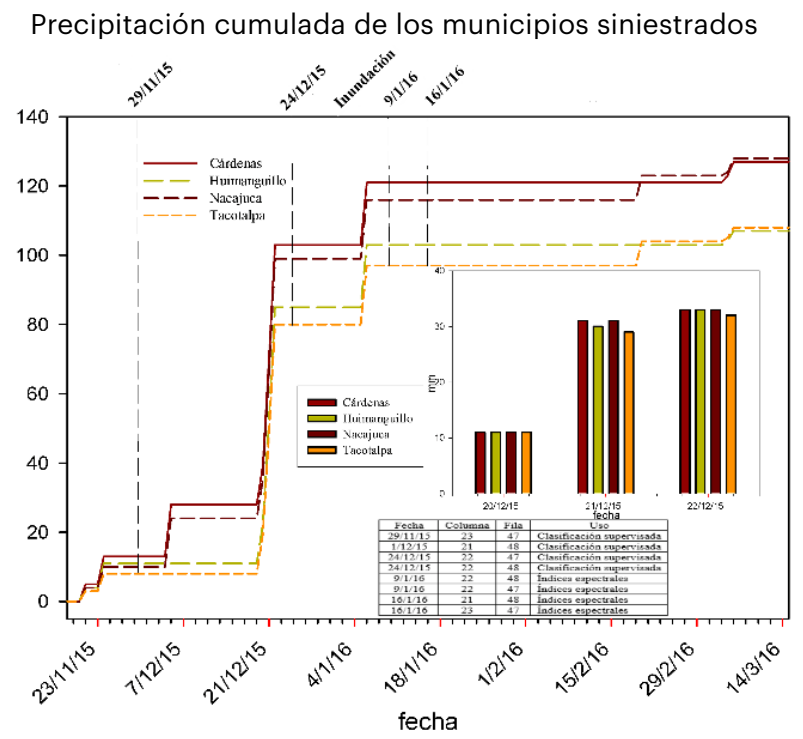

Fuente: Elaboración propia con información de la Base de datos diaria de Daymet. 


\section{Pre procesamiento de la información}

Se emplearon imágenes satelitales Landsat 8, convirtiendo los números digitales (ND) a valores de reflectancia de las bandas por imagen (Zanter, 2016). A su vez se hizo una corrección atmosférica. Este proceso se realizó en TerrSet (TerrSet versión 18.31, 2018) con el Cos(t) Model (Chavez, 1996). Se eliminaron las nubes de las imágenes, realizándose mediante una clasificación de nubes y terreno en general de la banda Quality Assessment Band (QAB). Esto fue hecho por medio de la herramienta de Landsat QA ArcMap Toolbox (ArcMap versión 10.2.2, 2014). Ya obtenidas las imágenes procesadas resultantes, se sumaron para conocer la información disponible para el periodo de estudio. La banda resultante QAB de cada imagen se multiplicó con el resto de las bandas con el fin de que las bandas tuvieran valores de 0 en donde se encontraban nubes.

\section{Identificación de cultivos de maíz}

Las imágenes satelitales Landsat 8 se segregaron a nivel municipal como lo recomienda Jia et al. (2014) para realizar la clasificación supervisada. Se realizó a través de la combinación de las bandas SWIR 1, Green y SWIR 2, y utilizando los valores de reflectancia (Skakun et al., 2016; Teferi et al., 2010). Para la identificación de los cultivos de maíz, se digitalizaron los sitios de entrenamiento con poligonales obtenidas de cultivos de maíz por medio de las zonas de cultivo identificadas de las cartas topográficas de INEGI escala 1:50,000, para posteriormente verificar dichas zonas con las imágenes Street View de Google Maps para las fechas de cultivo de maíz en esa zona. Asimismo, se identificaron otras cubiertas como: agua, zonas abandonadas, pantano, manglar, urbano, zonas inundadas, cultivos de caña y palma, entre otros. Cabe señalar que para cada municipio fueron diferentes las coberturas digitalizadas, debido a la heterogeneidad espacial de la región. Finalmente, se aplicó el clasificador Maximum likelihood con una probabilidad de muestra (Sample).

Para la evaluación y validación de la precisión de las clasificaciones, se usaron los sitios de entrenamiento como los puntos de referencia, ya que estos sitios fueron digitalizados con base en las poligonales obtenidas de Google Maps y de las cartas topográficas. Los métodos utilizados para la evaluación y validación fueron la precisión general (Overall Accuracy) (Stehman, 1997), el coeficiente kappa (Cohen, 1960), la exactitud del productor (Producer's Accuracy) y la exactitud del usuario (User's Accuracy) (Congalton, 1991).

\section{Identificación de zonas inundadas}

A fin de identificar las áreas inundadas se calcularon los índices espectrales de agua NDWI y MNDWI, vegetación NDVI, humedad VSDI (Visible and Shortwave infrared Drought Index) y la banda NIR a los cuales se les hizo una reclasificación para discriminar lo que es agua de lo que no lo es, debido a que existieron valores menores a 0 en dónde se sabía que era agua, como lo menciona (Du et al., 2012).

De acuerdo con (Xu, 2006), el NDWI y MNDWI tienen las siguientes ecuaciones:

$$
N D W I=\frac{\text { Green }-N I R}{\text { Green }+N I R}
$$




$$
M N D W I=\frac{\text { Green }-M I R}{\text { Green }+M I R}
$$

Donde NDWI es el Índice de Diferencia de Agua Normalizado, Green es la reflectancia de la banda verde, NIR es la reflectancia de la banda de infrarrojo cercano. MNDWI es el Índice de Diferencia de Agua Normalizado Modificado, MIR es la reflectancia de la banda del infrarrojo medio.

De acuerdo con (Gilabert, González-Piqueras \& García-Haro, 1997), la ecuación del NDVI es la siguiente:

$$
N D V I=\frac{N I R-R e d}{N I R+R e d}
$$

Donde NDVI es el Índice de Diferencia de Vegetación Normalizada, Red es la reflectancia de la banda roja, NIR es la reflectancia de la banda de infrarrojo cercano.

De acuerdo con (Zhang et al., 2013), la ecuación del VSDI es la siguiente:

$$
V S D I=1-[(S W I R-B l u e)+(\text { Red }- \text { Blue })]
$$

Donde VSDI es el Índice de Sequía infrarroja visible y de Onda Corta, SWIR es la reflectancia del infrarrojo de onda corta, Blue es la reflectancia de la banda azul y Red es la reflectancia de la banda roja.

\section{Estimaciones económicas por daños en zonas agrícolas}

Con las zonas de agua obtenidas mediante los índices espectrales y los cultivos de maíz de las clasificaciones supervisadas se procedió a extraer los valores de los índices espectrales para los cultivos de maíz por municipio, con el fin de ver los cambios en las reflectancias e identificar dónde fueron esos cambios, pudiendo así identificar superficies siniestradas de maíz por inundaciones. Esto se hizo a través de la determinación de umbrales para cada índice. Debido a que no existe una forma de saber si lo que se inundó verdaderamente se inundó, los diferentes índices utilizados para determinar las zonas inundadas se cruzaron para evaluar su consistencia en cuanto a la detección de zonas inundadas de maíz. Esta consistencia se evaluó con el coeficiente kappa y el User's Accuracy.

Identificadas las superficies siniestradas por inundaciones en las imágenes de satélite y en las bases de datos del SIAP se calcularon las estimaciones económicas, haciéndose de manera lineal y de manera espacial, con la finalidad de comparar ambas estimaciones con el índice de eficiencia de Nash-Sutcliffe (ENS) (Nash \& Sutcliffe, 1970), ya que, las bases de datos del SIAP no incluyen la información de la causa por la cual fueron siniestrados los cultivos de maíz.

Para reportar los daños, se estandarizaron los valores en USD en precios de 2016 utilizando tipos de cambio ajustados por paridad de poder adquisitivo (PPA) (World Bank, 2020), siendo para el caso de México para el año en estudio de 2.21. Asimismo, se tomó como punto de referencia el valor del dólar para el mes de marzo de 2016, siendo este de 18.33 pesos mexicanos. 


\section{Estimaciones económicas espaciales por daños en cultivos de maíz}

Fueron necesarias las superficies de maíz municipales obtenidas mediante la clasificación supervisada, para obtener las superficies de maíz siniestradas por inundación, las cuales fueron calculadas por medio de la aplicación de los umbrales de los índices espectrales y banda NIR y que coincidieran con las superficies de maíz siniestradas reportadas oficialmente por el SIAP; también fueron necesarios el valor y rendimiento de las bases de datos del SIAP, el factor de impacto para maíz y modificar la fórmula de Förster. La fórmula para la estimación de manera espacial es:

$$
E_{D}=M_{V} \times Y \times A_{C I} \times D_{I}
$$

Dónde $E D_{\text {Pixel }}$ es la estimación del daño por píxel $(\$), M_{v}$ es el valor del Mercado $(\$ / t), Y$ es el

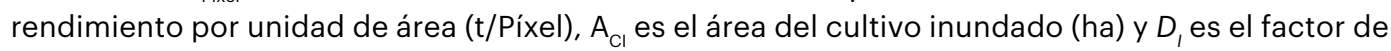
impacto del daño (\%). El área de cada píxel equivale a $900 \mathrm{~m}^{2}$ (0.09 ha), debido a que la resolución de pixel es de $30 \mathrm{~m} \times 30 \mathrm{~m}$ en Landsat 8 . El factor de impacto asignado para el cálculo de las estimaciones económicas lineales y espaciales fue de $80 \%$, debido al mes y la duración que tuvo la inundación en los cultivos.

\section{Estimaciones económicas lineales por daños en cultivos de maíz}

Se hizo con los datos reportados oficialmente por el SIAP (SAGARPA, 2018). Los datos necesarios fueron las superficies de maíz sembradas, cosechadas y siniestradas, así como su valor económico y rendimiento de estas. La escala está en hectáreas (ha). Con esta información se procedió a aplicar el método de Förster (Förster et al., 2008) para estimar los daños tangibles directos para el cultivo del maíz a causa de las inundaciones. La ecuación del método es la siguiente:

$$
E_{D}=M_{V} \times Y \times A \times D_{I}
$$

Dónde $E_{D}$ es la estimación del daño $(\$), M_{V}$ es el valor del Mercado $(\$ / t), Y$ es el rendimiento

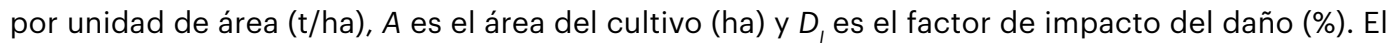
valor de mercado se calcula por el rendimiento total de un cultivo cosechado multiplicado por su precio de venta, difiere de una región a otra ya que el rendimiento del cultivo depende de las condiciones climáticas, del suelo, y del tipo de prácticas de manejo agrícola utilizadas. El factor de impacto de daño depende del tipo de cultivo, el mes o los meses que ocurren las inundaciones y la duración de la inundación de acuerdo con (Landesamt für Umwelt und Geologie (LfUG), 2005).

\section{Resultados y discusión}

\section{Identificación de cultivos}

En la Figura $\mathrm{N}^{\circ} 3$ se muestran las clasificaciones de los municipios siniestrados y en el Cuadro №1 las hectáreas de maíz, maíz siniestrado, los valores de reflectancias usados, así como las diversas precisiones de las clasificaciones (precisión general, exactitud del productor, exactitud del usuario y kappa). 
Figura $\mathrm{N}^{\circ} 3$.

Clasificación supervisada de los municipios siniestrados (A-Cárdenas, B-Huimanguillo, C-Nacajuca y D-Tacotalpa)
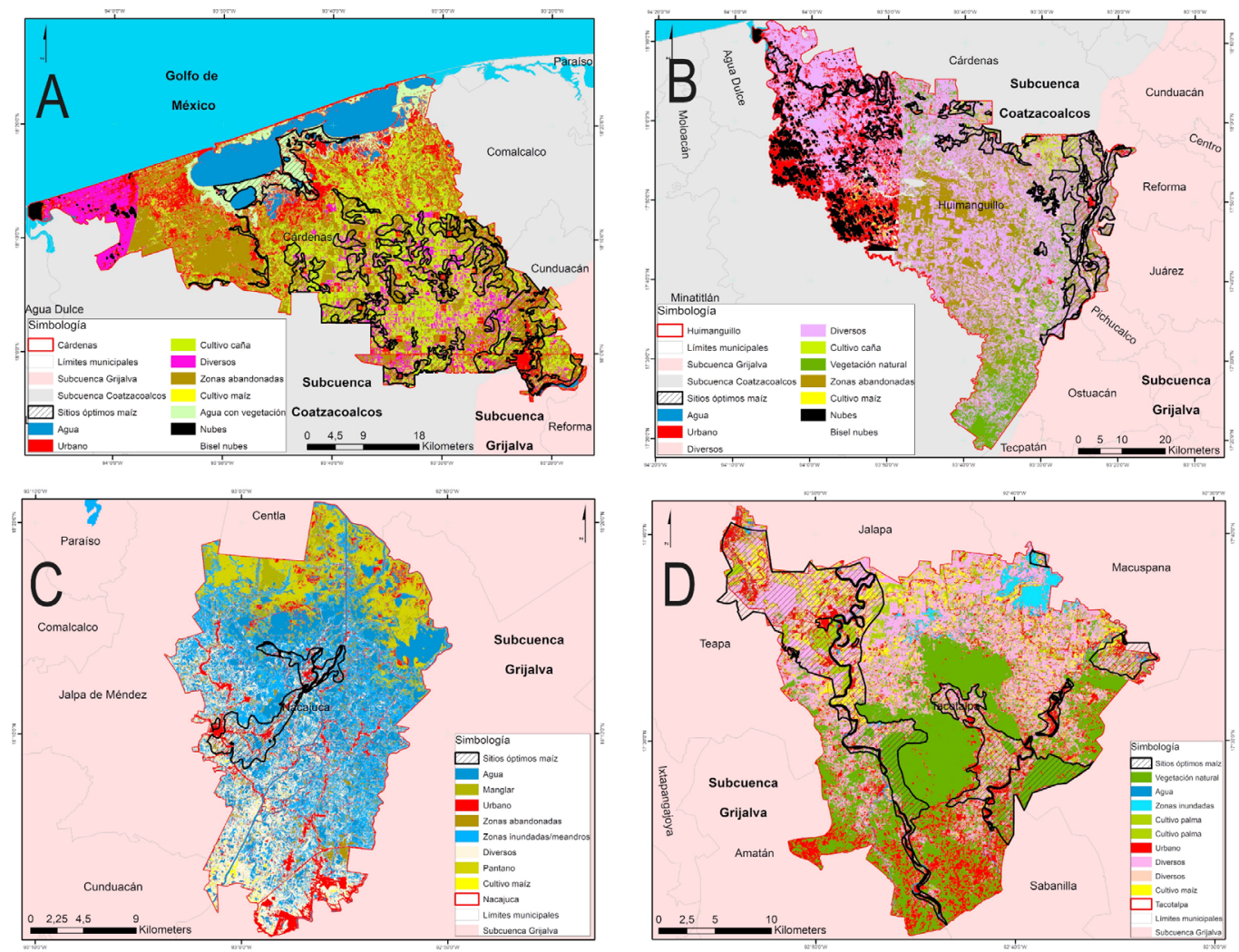

Fuente: Elaboración propia con base en imágenes de satélite Landsat 8 y cartas topográficas 1:50,000 de INEGI.

Para el municipio de Cárdenas se identificaron 9 clases. En Huimanguillo se obtuvieron 10 clases, incluyendo en la clase "diversos" a varios tipos de cultivos que no fueron tan notorios (cultivo de caña). En el caso de Nacajuca se tuvieron 8 clases, encontrándose cubierto por un $60 \%$ de agua, a su vez el norte del municipio se encuentra cubierto por manglar y pantano. Finalmente, en Tacotalpa se identificaron 9 clases, el cual presenta zonas pantanosas en la parte noreste. En los cuatro municipios se tuvieron sitios óptimos para el cultivo de maíz, en el (Cuadro N¹) se encuentran las superficies de maíz obtenidas por medio de la clasificación y las reportadas (SIAP). El índice Kappa para todos los municipios estuvo por encima de 0.9, mientras que en exactitud del productor se obtuvo un valor de 63.07 en Cárdenas y en exactitud del usuario se tuvieron valores en Cárdenas de 32.03 y en Nacajuca de 15.45, estos valores bajos pueden deberse debido a que la imagen se encontraba cubierta por nubes afectando los resultados de las clasificaciones. En los casos de Cárdenas y Huimanguillo, se tienen dos valores en las pruebas de precisión, debido a que se usaron dos imágenes para realizar sus clasificaciones, por lo cual, en Cárdenas los valores de 99.81 , 0.994, 100 y 96.15 y en Huimanguillo de 94.62, 0.902, 100 y 54.09 corresponden a la 
escena 022 048; mientras que los valores de 98.14, 0.97, 63.07 y 32.03 para el municipio de Cárdenas y $91.59,0.881,88.88$ y 82.75 para Huimanguillo son de la escena 023047 .

Cuadro $N^{\circ} 1$.

Superficie siniestrada estimada, reportada y nivel de precisión de la metodología para estimar

\begin{tabular}{|c|c|c|c|c|c|c|c|c|c|}
\hline Municipio & Algoritmo & Probabilidad & \begin{tabular}{|c|} 
Valores \\
reflectancia \\
(637)
\end{tabular} & $\begin{array}{l}\text { Superficie } \\
\text { (ha) SIAP }\end{array}$ & $\begin{array}{c}\text { Superficie (ha) } \\
\text { clasificación } \\
\text { supervisada }\end{array}$ & $\begin{array}{c}\text { Precisión } \\
\text { general }\end{array}$ & Kappa & $\begin{array}{c}\text { Exactitud } \\
\text { del } \\
\text { productor } \\
\text { (maíz) }\end{array}$ & \begin{tabular}{|c} 
Exactitud \\
del \\
usuario \\
(maíz)
\end{tabular} \\
\hline \multirow[b]{2}{*}{ Cárdenas } & \multirow[b]{2}{*}{$\begin{array}{l}\text { Maximum } \\
\text { Likelihood }\end{array}$} & \multirow[b]{2}{*}{ Sample } & R 0.30-0.34 & \multirow[b]{2}{*}{3.218} & \multirow[b]{2}{*}{3,189} & 99.81 & 0.994 & 100 & 96.15 \\
\hline & & & \begin{tabular}{|c|}
$G$ 0.07-0.10 \\
B $0.14-0.16$ \\
\end{tabular} & & & 98.14 & 0.97 & 63.07 & 32.03 \\
\hline \multirow{2}{*}{$\begin{array}{l}\text { Huiman- } \\
\text { guillo }\end{array}$} & \multirow{2}{*}{$\begin{array}{l}\text { Maximum } \\
\text { Likelihood }\end{array}$} & \multirow[b]{2}{*}{ Sample } & \begin{tabular}{|l|}
$R 0.30-0.34$ \\
\end{tabular} & \multirow[b]{2}{*}{5,380} & \multirow[b]{2}{*}{5,455} & 94.62 & 0.902 & 100 & 54.09 \\
\hline & & & \begin{tabular}{|c|}
$G$ 0.07-0.10 \\
B $0.14-0.16$ \\
\end{tabular} & & & 91.59 & 0.881 & 88.88 & 82.75 \\
\hline Tacotalpa & $\begin{array}{l}\text { Maximum } \\
\text { Likelihood }\end{array}$ & Sample & \begin{tabular}{|c|} 
R $0.30-0.34$ \\
$G 0.07-0.10$ \\
$B \quad 0.14-0.16$ \\
\end{tabular} & 4,690 & 4,801 & 98.4 & 0.989 & 100 & 100 \\
\hline Nacajuca & $\begin{array}{l}\text { Maximum } \\
\text { Likelihood }\end{array}$ & Sample & \begin{tabular}{|l|l|}
$R$ & $0.37-0.40$ \\
$G \quad 0.06-0.08$ \\
$B$ & $0.36-0.38$ \\
\end{tabular} & 610 & 631 & 95.58 & 0.967 & 96.96 & 15.45 \\
\hline
\end{tabular}

Fuente: Elaboración propia.

Como se puede observar (Cuadro $\mathrm{N}^{\circ} 1$ ), las superficies de maíz reportadas de manera oficial por el SIAP y las obtenidas por la vía de la clasificación supervisada a nivel municipal utilizando el algoritmo de Maximum Likelihood y la probabilidad de Sample no varían demasiado. Asimismo, se observan resultados por encima del 90\% (Overall) y 0.9 (Kappa), lo que quiere decir que fueron buenas las clasificaciones.

\section{Identificación de zonas inundadas}

La identificación de las zonas inundadas se realizó por medio de la aplicación de los índices espectrales de agua, vegetación, humedad y la banda NIR (Figura N4).

En el municipio de Cárdenas (Figura N4-A) los índices de agua (MNDWI y NDWI), el índice NDVI y la banda NIR son los que mejor discriminan las zonas inundadas o cubiertas por agua, mientras que el índice VSDI identifica como inundadas las zonas de vegetación en donde no hay agua, esto por la cantidad de humedad que tiene la vegetación. En (Figura N4-B) todos los índices (MNDWI, NDWI, NDVI y VSDI) y la banda NIR discriminan bien las zonas inundadas de manera general, pero de manera específica son los índices NDVI, VSDI y banda NIR los mejores, ya que hay zonas cubiertas por vegetación donde hay agua que, si identifican, algo que los índices de agua no hacen. En (Figura $\mathrm{N}^{\circ} 4-\mathrm{C}$ ) todos los índices identifican diferentes zonas de inundación, teniendo al NDVI y a la banda NIR con resultados similares, pero los otros tres índices (MNDWI, NDWI y VSDI) discriminan más superficie que otros, como es el caso del VSDI que identifica casi todo el municipio cubierto por agua, siguiéndole el MNDWI en la parte central y teniendo al final al NDWI con menor superficie identificada como inundada. En (Figura N4-D) los índices MNDWI y NDWI junto con el índice NDVI son los que mejor discriminan las zonas inundadas o cubiertas por agua, mientras 


\section{Figura $\mathrm{N}^{\circ} 4$}

Índices espectrales (1-MNDWI, 2-NDWI, 3-NDVI, 4-VSDI) y banda NIR (5) de los municipios siniestrados (A-Cárdenas, B-Huimanguillo, C-Nacajuca y D-Tacotalpa).
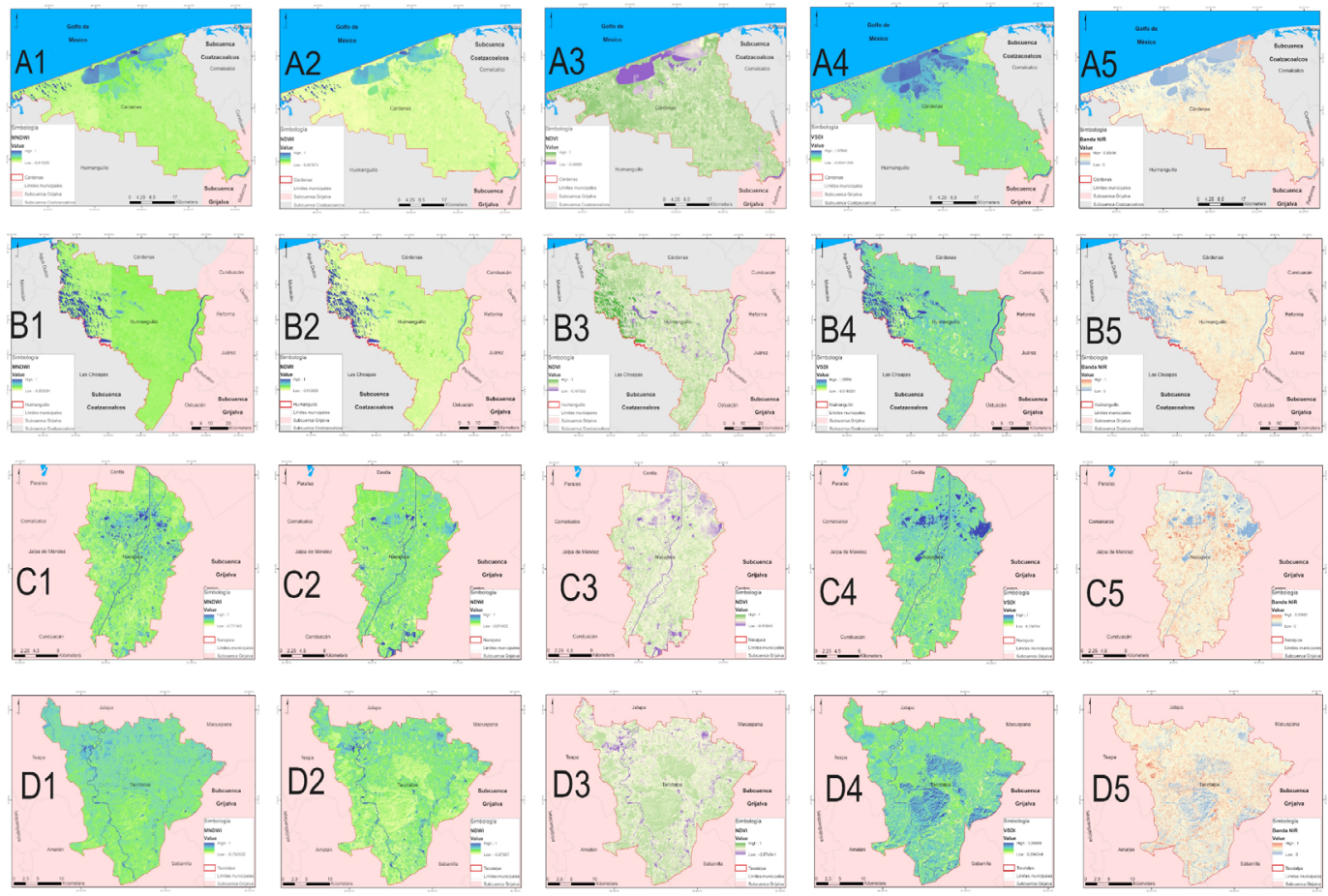

Fuente: Elaboración propia.

que la banda NIR y el índice VSDI identifican principalmente el río principal, pero también zonas de vegetación en donde no hay agua, esto por la cantidad de humedad que tiene la vegetación al momento de la toma de la imagen de satélite.

\section{Estimaciones económicas}

Diversos autores como (Kelly \& Gontz, 2018; Mohammadi, Costelloe \& Ryu, 2017; Noori et al., 2016) recomiendan el uso de la aplicación de diferentes índices espectrales como son el MNDWI, NDVI e incluso la banda del infrarrojo cercano para la identificación de zonas inundadas, tanto para imágenes Landsat como para MODIS.

Debido a que no se contó con datos de la altura de la lámina, para la obtención de las estimaciones económicas espaciales se usaron umbrales para los valores de los índices y la banda NIR para cada municipio (Cuadro $\mathrm{N}^{\circ} 2$ ). 


\section{Cuadro $\mathrm{N}^{\circ} 2$}

Umbrales espectrales de los índices empleados para caracterizar las zonas de inundación

\begin{tabular}{|l|c|c|c|c|c|}
\hline \multicolumn{1}{|c|}{ Municipio } & MNDWI & NDWI & NDVI & NIR & VSDI \\
\hline Cárdenas & $-0.502-0.999$ & $-0.5878-0.999$ & $0.8026-0.999$ & $0.55491-0.999$ & $0.8014-0.999$ \\
\hline Huimanguillo & $-0.4666-0.999$ & $-0.5849-0.999$ & $0.8585-0.999$ & $0.6451-0.999$ & $0.8290-0.999$ \\
\hline Nacajuca & $-0.6177-0.999$ & $-0.6996-0.999$ & $0.8083-0.999$ & $0.6435-0.999$ & $0.6589-0.999$ \\
\hline Tacotalpa & $-0.5531-0.999$ & $-0.6838-0.999$ & $0.8374-0.999$ & $0.60671-0.999$ & $0.7693-0.999$ \\
\hline
\end{tabular}

Fuente: Elaboración propia.

Se recomienda que los umbrales sean dinámicos, es decir, los umbrales planteados para la identificación de zonas inundadas de cultivo de maíz son solamente aplicables para la fecha exacta en que fueron obtenidos (diciembre 2015, enero 2016). Esto debido a las características climáticas, naturales y antrópicas que se presentan en el Estado de Tabasco, pueden variar ampliamente de un mes a otro, lo cual ocasiona que no puedan ser usados los mismos umbrales y por consecuencia se tengan que determinar umbrales para cada estudio de esta índole.

La determinación de umbrales ha sido usada en diferentes trabajos como los de (Acharya et al., 2017; Boschetti et al., 2014; Morsy et al., 2016), en los cuales la importancia del uso de estos umbrales es conocer mediante la aplicación de umbrales diferentes tipos de coberturas sin necesidad de realizar otra clase de proceso en las imágenes. A su vez, (Ji, Zhang \& Wylie, 2009) en su trabajo sobre la utilización de umbrales menciona que los umbrales en muchas ocasiones son dinámicos por diversos factores como es el tipo de cobertura, su color, sedimentos, vegetación cercana, hora del día de la imagen, así como el ángulo en que fue tomada la imagen.

Con la determinación de los umbrales, se tienen los siguientes resultados para las estimaciones económicas espaciales que se presentan (Cuadro N³). En la (Figura $N^{\circ} 5$ ) se observan los cultivos de maíz, que están divididos en valores, que es, la concordancia que hay entre los índices para detectar las mismas superficies siniestradas. El valor de 0 son las hectáreas que no fueron siniestradas, 1 son las hectáreas que fueron siniestradas mediante un índice, 2 son las hectáreas que fueron siniestradas con dos índices, 3 son las hectáreas que fueron siniestradas por tres índices y 4 son las hectáreas que fueron siniestradas por cuatro índices, teniendo como los resultados más cercanos a lo reportado el cruce realizado con 2 índices.

En la Figura N5-A se muestran los cultivos de maíz en el municipio de Cárdenas. Se puede observar que una parte de los cultivos de maíz siniestrados se encuentra en las cercanías de los ríos. Esto concuerda con los reportes de la región donde se menciona que los cultivos más afectados son debido al desbordamiento de los ríos. El cruce que muestra valores más altos tanto en kappa como en user's accuracy es NDVI-B5. La Figura N 5-B muestra el cultivo de maíz en el municipio de Huimanguillo, las superficies siniestradas tienen relación con la red de drenaje debido a que gran parte de los cultivos se localizan en las cercanías de ríos, ocasionando que éstos se desborden por las lluvias y siniestren las áreas de cultivos. El cruce que muestra valores más altos en kappa y en user's accuracy es MNDWI-NDWI. La Figura $N^{\circ}$ 5-C muestra las áreas de cultivo de maíz en el municipio de Nacajuca, en este municipio se cuenta con una vasta red de cuerpos de agua y ríos, lo cual hace que sea un municipio con riesgo por inundación en las zonas de cultivos (Domínguez, 2019; Instituto de Protección Civil del Estado de Tabasco, 2016), muchos de estos ríos 
Figura $\mathrm{N}^{\circ} 5$.

Superficies siniestradas de los municipios
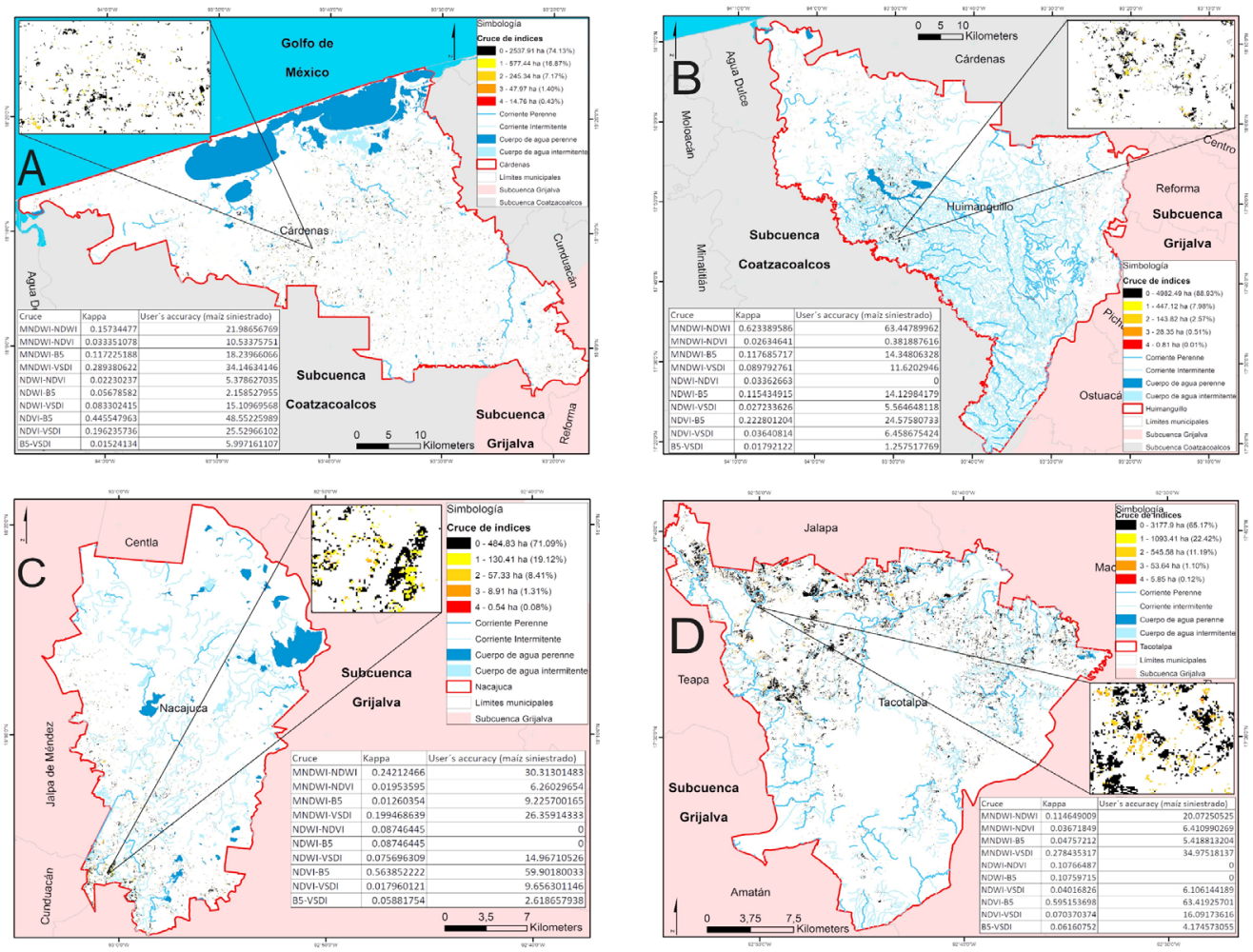

Fuente: Elaboración propia.

y cuerpos de agua son de carácter intermitentes, lo que los hace más peligrosos, debido a que la población se confía que su nivel de agua es bajo o inexistente, pero que en temporada de lluvias sus niveles ascienden, haciendo que muchas veces estos se desborden y lleguen a siniestrar cultivos en muy corto tiempo. Para este municipio el cruce que muestra valores más altos en kappa y en user's accuracy es NDVI-B5. En la Figura $N^{\circ} 5$-D se muestra el cultivo de maíz en el municipio de Tacotalpa. La gran mayoría de los ríos que tiene el municipio se localizan principalmente en las partes noroeste y noreste, que a su vez también son los lugares en donde existen más cultivos de maíz, haciendo propensas estas áreas a que sean siniestradas por el desbordamiento de estos ríos. El cruce que muestra valores más altos en kappa y en user's accuracy es el NDVI-B5.

Una vez establecidas las zonas afectadas por inundación, en el Cuadro $\mathrm{N}^{\circ} 3$, se muestran las estimaciones económicas espaciales, así como las lineales realizadas por medio de las bases de datos del SIAP. En este cuadro se encuentra la información adicional que se utilizó para el cálculo de ambas estimaciones, que son el valor de rendimiento, valor por tonelada cosechada y el factor de impacto, los cuales son datos ya estandarizados por la SAGARPA, donde el municipio de Tacotalpa es el municipio con mayor rendimiento ( $1.9 \mathrm{t} / \mathrm{ha})$, mientras que Nacajuca es el de menor rendimiento ( $0.9 \mathrm{t} / \mathrm{ha}$ ). El factor de rendimiento está en función del mes de la inundación, así como la duración de ésta. Debido a que la inundación ocurrió en diciembre y duró más de 11 días 
el factor de impacto fue de 0.80. Para las superficies siniestradas, se obtuvieron los valores por medio de la determinación y aplicación de umbrales para cada índice que se usó (MNDWI, NDWI, NDVI, VSDI) y la banda NIR en los cuatro municipios siniestrados.

Es importante señalar que en el presente trabajo existe incertidumbre en las superficies siniestradas espaciales, puesto que no se sabe si en verdad fueron las superficies siniestradas reportadas de manera lineal por el SIAP. Lo anterior, debido a que los datos oficiales no cuentan con coordenadas de las áreas siniestradas para poder validar las superficies espaciales siniestradas. Sin embargo, al comparar de groso modo los daños reportados por el SIAP a nivel municipal, contra el daño estimado de manera espacial se encontró una eficiencia de 0.95, significando que se tuvo una coincidencia casi perfecta con los datos observados, teniendo como datos observados lo reportado por el SIAP.

Cuadro $\mathrm{N}^{\circ} 3$

Estimaciones económicas

\begin{tabular}{|c|c|c|c|c|c|c|c|c|}
\hline Municipio & $\begin{array}{c}\text { Rendimiento } \\
\text { (t/ha) }\end{array}$ & $\begin{array}{c}\text { Factor de } \\
\text { impacto } \\
\text { (\%) }\end{array}$ & $\begin{array}{c}\text { Valor por } \\
\text { tonelada } \\
\text { cosechada } \\
\text { (USD/t en } \\
\text { PPA) }\end{array}$ & $\begin{array}{c}\text { Daño por } \\
\text { ha (USD/ha } \\
\text { en PPA) }\end{array}$ & $\begin{array}{c}\text { Superficie } \\
\text { (ha) }\end{array}$ & $\begin{array}{c}\text { Eaño total } \\
\text { (USD en } \\
\text { PPA) }\end{array}$ & $\begin{array}{c}\text { Superficie } \\
\text { (ha) }\end{array}$ & $\begin{array}{c}\text { Laño total } \\
\text { (USD en } \\
\text { PPA) }\end{array}$ \\
\hline Cárdenas & 1.49 & 0.80 & 472.14 & 563.58 & 245.34 & $138,269.33$ & 255.00 & $143,713.54$ \\
\hline Huimanguillo & 1.50 & 0.80 & 449.23 & 539.27 & 143.82 & $77,558.00$ & 165.00 & $88,979.77$ \\
\hline Nacajuca & 0.90 & 0.80 & 440.19 & 317.25 & 57.33 & $18,188.22$ & 55.00 & $17,449.01$ \\
\hline Tacotalpa & 1.95 & 0.80 & 467.06 & 728.57 & 545.58 & $397,492.47$ & 475.00 & $346,070.10$ \\
\hline
\end{tabular}

*ha= hectáreas; ${ }^{*} \mathrm{USD}=$ Dólares americanos, ${ }^{*} \mathrm{t}=$ tonelada, ${ }^{* P P A}=$ Paridad de Poder Adquisitivo Fuente: Elaboración propia.

Para las estimaciones económicas calculadas se obtuvieron los mismos resultados, tanto para las espaciales, como para las económicas, (Cuadro $N^{\circ} 3$ ). Tacotalpa fue el municipio que tuvo más hectáreas siniestradas ( 475 ha lineales y 545.58 ha espaciales), mientras que Nacajuca tuvo menor cantidad de superficie siniestrada (55 ha lineales y 57.33 ha espaciales). Los daños por hectárea (USD/ha en PPA) para los municipios de Cárdenas y Huimanguillo fueron de 563.58 USD/ha en PPA y 539.27 USD/ha en PPA respectivamente, mientras que en Nacajuca fue de 317.25 USD/ha en PPA y en Tacotalpa de 728.57 USD/ha en PPA. Se entiende que los resultados son diferentes, principalmente en los municipios de Nacajuca y Tacotalpa, debido a que, sus factores de rendimiento y el número de hectáreas afectadas son diferentes, caso contrario con los municipios de Cárdenas y Huimanguillo, en donde, sus factores de rendimiento y superficie siniestrada son similares.

De acuerdo con lo reportado por (Suárez et al., 2007), el daño con el cultivo de maíz para el año 2003 para la Cuenca Alta del Río Lerma, fueron de 522.25 USD/ha en PPA en 4,489.8 ha, resultados similares a los obtenidos en Cárdenas y Huimanguillo, en el daño, más no en las hectáreas. Mientras que (Tapia-Silva et al., 2011) reportó un daño al maíz por inundaciones en Alemania para el año 2002 de 323.75 USD/ha en PPA en 1,437 ha y Förster et al. (2008) lo hizo para la misma fecha, lugar y hectáreas de 244.75 USD/ha en PPA; si bien, no son resultados iguales a los reportados en Nacajuca, si existe similitud, en cuanto al daño, a pesar que es en otro país. Se puede 
entender que, los daños obtenidos en la presente investigación son similares a los realizados en otras partes del mundo.

Comparando los resultados en hectáreas afectadas, si, es mayor el daño que se obtuvo en los municipios del Estado de Tabasco, puesto que son menos hectáreas que en los estudios antes mencionados. Esto tiene que ver con el rendimiento que se tiene en cada lugar para determinado cultivo, ya que como se observa en el Cuadro $\mathrm{N}^{\circ} 3$, los rendimientos para maíz en el ciclo de otoño-invierno son muy bajos en comparación con otros estados como el Estado de México, con rendimientos promedio de $4.5 \mathrm{t} / \mathrm{ha}$, Jalisco con un promedio de $6.5 \mathrm{t} / \mathrm{ha}$ y Sinaloa con promedio de $9.5 \mathrm{t} /$ ha (SAGARPA, 2018).

Es importante resaltar que, en el caso de los resultados obtenidos en este estudio, el factor de rendimiento por hectárea, es en gran medida, una parte fundamental para obtener el daño total, ya que como se pudo observar (Cuadro $N^{\circ} 3$ ), son diferentes, aunque se encuentren en el mismo Estado, por lo cual es imprescindible considerarlo al momento de realizar estimaciones económicas en algún tipo de cultivo (Rahman \& Di, 2020; Vozinaki et al., 2015; Van der Sande, De Jong \& De Roo, 2003).

\section{Conclusión}

Las inundaciones ocurridas en diciembre del 2015 pudieron haber sido a causa de los escurrimientos de las microcuencas de la parte alta de la Subcuenca del Río Grijalva, debido a que las precipitaciones en toda la región fueron homogéneas.

El uso de imágenes de Street View de Google para detectar cultivos de maíz ayudó a obtener buenos resultados en cuanto a la precisión de su clasificación, teniendo porcentajes en Precisión general y en kappa por encima del $90 \%$.

Se puede decir, que, los índices que detectaron mejor los polígonos de inundación fueron los de agua (MNDWI y NDWI), seguidos por el de vegetación (NDVI) y la banda NIR, y finalmente el índice de humedad (VSDI). A pesar de lo anterior, es recomendable el uso de varios índices puesto que la precisión de estos dependerá de las características particulares de la zona de estudio.

Las evaluaciones de pérdidas en rendimiento y en términos monetarios brindan información más detallada en comparación con informes simples de superficie inundada. Sin embargo, es necesario que los datos históricos de rendimiento y el precio de los cultivos estén disponibles. Además, los datos sobre precios y rendimiento de cultivos específicos son muy escasos a nivel local. Aunque hay muchas formas de informar la pérdida de cultivos por inundación, la opción de informar los daños completamente depende del propósito del estudio y de la audiencia. Para este estudio los resultados de daños por hectárea estuvieron entre los 728.57 USD/ha en PPA y los 317.25 USD/ha en PPA los cuales concuerdan con las estimaciones realizadas en otros países.

A pesar que los resultados obtenidos son similares a otros resultados de diferentes partes del mundo, no hay que olvidar, que en la investigación se plantea el ciclo productivo de maíz de 
otoño-invierno, siendo este de autoconsumo y no de exportación, por lo cual toma un sentido especial el tener estos daños, puesto que, son cultivos que en la gran mayoría de los casos no se encuentran asegurados, por lo cual, son pérdida total y que esta pérdida de cultivo impide que las familias que dependen de él como parte de su alimentación, tengan que buscar otras formas de reemplazar el maíz en su alimentación.

La presente investigación plantea una forma diferente para estimar pérdidas económicas espaciales por inundaciones mediante la determinación zonas dañadas usando umbrales dinámicos para distintos índices espectrales. Puesto que primordialmente el uso de estos índices es para la identificación de cuerpos de agua, vegetación u otras coberturas de manera muy general. Por lo cual, el plus de esta investigación fue dar una nueva aplicación a estos índices, obteniendo buenos resultados. Sin embargo, debido a la incertidumbre en cuanto a la concordancia de los valores reportados por el SIAP y las estimaciones espaciales se recomienda que en estudios futuros se cuente con datos más precisos de las superficies dañadas (e.g. polígonos de inundación, tirantes de inundación o coordenadas).

\section{Referencias}

ACHARYA, T. D., LEE, D. H., YANG, I. T., \& LEE, J. K. Identification of water bodies in a Landsat 8 OLI image using a J48 decision tree. Sensors. 2016. Vol. 16, № 7, p. 1075.

ACHARYA, T. D., SUBEDI, A., YANG, I. T., \& LEE, D. H. Combining water indices for water and background threshold in landsat image. In: Multidisciplinary Digital Publishing Institute Proceedings. 2017. p. 143.

AGUILAR, A., \& BEDOYA, G. Creación, actualización y/o homogeneización de inventarios de desastres por eventos históricos y cotidianos a nivel de la Subregión Andina. Informe de análisis inventario de pérdidas por desastres Vers. 2006. Vol. 1.

ARCMAP VERSIÓN 10.2.2. ESRI. 2014.

BALICA, S.F., POPESCU, I., BEEVERS, L., \& WRIGHT, N.G. Parametric and physically based modeIling techniques for flood risk and vulnerability assessment: A comparison. Environmental Modelling \& Software. March 2013. Vol. 41, p. 84-92.

BARÓ SUÁREZ, J. E., DìAZ DELGADO, C., CALDERÓN ARAGÓN, G., ESTELLER ALBERICH, M. V., CADENA VARGAS, E., \& FRANCO PLATA, R. Metodología para la valoración económica de daños potenciales tangibles directos por inundación. Universidad Autónoma del Estado de México, 2012.

BOSCHETTI, M., NUTINI, F., MANFRON, G., BRIVIO, P. A., \& NELSON, A. Comparative analysis of normalised difference spectral indices derived from MODIS for detecting surface water in flooded rice cropping systems. PloS one. 2014. Vol. 9, № 2, p. e88741. 
CHAVEZ, P. S. Image-based atmospheric corrections-revisited and improved. Photogrammetric engineering and remote sensing. 1996. Vol. 62, № 9, p. 1025-1035.

COHEN, J. A coefficient of agreement for nominal scales. Educational and psychological measurement. 1960. Vol. 20, No 1, p. 37-46.

CONGALTON, R. G. A review of assessing the accuracy of classifications of remotely sensed data. Remote sensing of environment. 1991. Vol. 37, № 1, p. 35-46.

CROW, H. A. Assessment of the FEMA HAZUS-MH 2.0 Crop Loss Tool Fremont County, lowa 2011. University of Southern California, 2014.

DÍAZ-BORREGO, L., \& RODRÍGUEZ-INFANTE, A. Evaluación del riesgo por inundación en la comunidad Pradera Alta, municipio Maracaibo, Venezuela. Minería y Geología. 2016. Vol. 32, № 3, p. 1-15.

DOF. Declaratoria de Desastre Natural por la ocurrencia de inundaciones en el Estado de Tabasco. 1999.

DOF. Declaratoria de Desastre Natural por la ocurrencia de inundaciones en el Estado de Tabasco. 2007.

DOMínGUEZ, A. A. Caso de Estudio: Control de Inundaciones en Tabasco. 2019.

DU, Z., BIN, L., LING, F., LI, W., TIAN, W., WANG, H., GUI, Y., SUN, B., \& ZHANG, X. Estimating surface water area changes using time-series Landsat data in the Qingjiang River Basin, China. Journal of Applied Remote Sensing. 2012. Vol. 6, № 1, p. 063609.

DUTTA, D., HERATH, S., \& MUSIAKE, K. A mathematical model for flood loss estimation. Journal of hydrology. 2003. Vol. 277, N¹-2, p. 24-49.

EM DAT (EMERGENCY EVENTS DATABASE). The OFDA/CRED international disaster database. 2016.

ESCUDER, I., MATHEU, E., \& CASTILLO, J. Análisis y evaluación de riesgos de inundación: estimación del impacto de medidas estructurales y no estructurales. Jornada CICCPV, Universidad Politécnica de Valencia, España. 2010.

FAO, IFAD. WFP (2015), The State of Food Insecurity in the World 2015. Meeting the 2015 international hunger targets: taking stock of uneven progress. Food and Agriculture Organization Publications, Rome. 2016.

FISHER, A., FLOOD, N., \& DANAHER, T. Comparing Landsat water index methods for automated water classification in eastern Australia. Remote Sensing of Environment. 2016. Vol. 175, p. 167-182.

FOOD AND AGRICULTURE ORGANIZATION OF THE UNITED NATIONS. The Impact of Natural Hazards and Disasters on Agriculture and Food Security and Nutrition: A Call for Action to Build Resilient Livelihoods. Food and Agriculture Organization of the United Nations, 2015. 
FÖRSTER, S., KUHLMANN, B., LINDENSCHMIDT, K. E. \& BRONSTERT, A. Assessing flood risk for a rural detention area. Natural Hazards and Earth System Science, 2008. Vol. 8, N² 2, p. 311-322.

FORTE, F., STROBL, R.O., \& PENNETTA, L. A methodology using GIS, aerial photos and remote sensing for loss estimation and flood vulnerability analysis in the Supersano-Ruffano-Nociglia Graben, southern Italy. Environmental Geology. 2006. Vol. 50, N 4, p. 581-594.

FRANCI, F., BOCCARDO, P., MANDANICI, E., ROVERI, E., \& BITELLI, G. Flood mapping using VHR satellite imagery: a comparison between different classification approaches. In : Earth Resources and Environmental Remote Sensing/GIS Applications VII. International Society for Optics and Photonics, 2016. p. 1000509.

GILABERT, M. A., GONZÁLEZ-PIQUERAS, J., \& GARCÍA-HARO, J. Acerca de los índices de vegetación. Revista de teledetección. 1997. Vol. 8, Nº 1, p. 1-10.

HERNÁNDEZ-URIBE, R., BARRIOS-PIÑA, H., \& RAMÍREZ, A. I. Análisis de riesgo por inundación: metodología y aplicación a la cuenca Atemajac. Tecnología y ciencias del agua. 2017. Vol. 8, $\mathrm{N}^{\circ} 3$, p. 5-25.

HOES, O., \& SCHUURMANS, W. Flood standards or risk analyses for polder management in the Netherlands. Irrigation and Drainage: The journal of the International Commission on Irrigation and Drainage. 2006. Vol. 55, Nº S1, p. S113-S119.

INEGI. 2018.

INSTITUTO DE PROTECCIÓN CIVIL DEL ESTADO DE TABASCO. 2016.

JI, L., ZHANG, L., \& WYLIE, B. Analysis of dynamic thresholds for the normalized difference water index. Photogrammetric Engineering \& Remote Sensing. 2009. Vol. 75, No 11, p. 1307-1317.

JIA, K., WEI, X., GU, X., YAO, Y., XIE, X., \& LI, B. Land cover classification using Landsat 8 operational land imager data in Beijing, China. Geocarto International. 2014. Vol. 29, N 8, p. 941-951.

JONGMAN, B., KREIBICH, H., APEL, H., BARREDO, J., BATES, P.D., FEYEN, L., GERICKE, A., NEAL, J., AERTS, J., \& WARD, P.J. Comparative flood damage model assessment: towards a European approach. Natural Hazards and Earth System Sciences (NHESS). 2012. Vol. 12, N 12, p. 3733-3752.

JUNG, Y., KIM, D., KIM, D., KIM, M., \& LEE, S. O. Simplified flood inundation mapping based on flood elevation-discharge rating curves using satellite images in gauged watersheds. Water. 2014. Vol. 6, N 5, p. 1280-1299.

KELLY, J. T., \& GONTZ, A. M. Using GPS-surveyed intertidal zones to determine the validity of shorelines automatically mapped by Landsat water indices. International journal of applied earth observation and geoinformation. 2018. Vol. 65, p. 92-104. 
LANDESAMT FÜR UMWELT UND GEOLOGIE (LFUG). Hochwasser in Sachsen - Gefahrenhinweisekarte, Sachsisches. 2005.

MERZ, B., KREIBICH, H., SCHWARZE, R. \& THIEKEN, A. Review article "Assessment of economic flood damage." Natural Hazards and Earth System Science. 18 August 2010. Vol. 10, Nº 8, p. 16971724.

MERZ, B., \& THIEKEN, A. H. Flood risk curves and uncertainty bounds. Natural Hazards. December 2009. Vol. 51, N³, p. 437-458.

MOGUEL, A. G., TEJEDA, A., \& GARCÍA PACHECO, V. H. Propuesta para la evaluación de riesgos por inundaciones urbanas: el caso de Xalapa (México). Publicaciones de la Asociación Española de Climatología. Serie A; 8. 2012.

MOHAMMADI, A., COSTELLOE, J. F., \& RYU, D. Application of time series of remotely sensed normalized difference water, vegetation and moisture indices in characterizing flood dynamics of large-scale arid zone floodplains. Remote sensing of environment. 2017. Vol. 190, p. 70-82.

MORSY, S., SHAKER, A., EL-RABBANY, A., \& LAROCQUE, P. E. Airborne Multispectral Lidar Data For Land-Cover Classification And Land/Water Mapping Using Different Spectral Indexes. ISPRS Annals of Photogrammetry, Remote Sensing \& Spatial Information Sciences. 2016. Vol. 3, No 3, p.

NASH, J. E., \& SUTCLIFFE, J. V. River flow forecasting through conceptual models part I-A discussion of principles. Journal of hydrology. 1970. Vol. 10, № 3, p. 282-290.

NOORI, N., KALIN, L., SEN, S., SRIVASTAVA, P., \& LEBLEU, C. Identifying areas sensitive to land use/ land cover change for downstream flooding in a coastal Alabama watershed. Regional Environmental Change. 2016. Vol. 16, Nº 6, p. 1833-1845.

64. RAHMAN, M. D., \& DI, L. A Systematic Review on Case Studies of Remote-Sensing-Based Flood Crop Loss Assessment. Agriculture. 2020. Vol. 10, № 4, p. 131.

RODRÍGUEZ GAVÍRIA, E. M. Diseño metodológico para la evaluación del riesgo por inundación a nivel local con información escasa. Escuela de Geociencias y Medio Ambiente. 2016.

SAGARPA. Atlas Agroalimentario. Servicio de Información Agroalimentaria y Pesquera. Primera. México, 2018.

SCAWTHORN, C., FLORES, P., BLAIS, N., SELIGSON, H., TATE, E., CHANG, S., MIFFLIN, E., THOMAS, W., MURPHY, J., \& JONES, C. HAZUS-MH flood loss estimation methodology. II. Damage and loss assessment. Natural Hazards Review. 2006. Vol. 7, № 2, p. 72-81.

SHARMA, T. P. P., ZHANG, J., KOJU, U. A., ZHANG, S., BAI, Y., \& SUWAL, M. K. Review of flood disaster studies in Nepal: A remote sensing perspective. International journal of disaster risk reduction. 2019. Vol. 34, p. 18-27. 
SINGH, A., \& SINGH, K. K. Satellite image classification using genetic algorithm trained radial basis function neural network, application to the detection of flooded areas. Journal of Visual Communication and Image Representation. 2017. Vol. 42, p. 173-182.

SINGH, K. K., \& SINGH, A. Identification of flooded area from satellite images using Hybrid Kohonen Fuzzy C-Means sigma classifier. The Egyptian Journal of Remote Sensing and Space Science. 2017. Vol. 20, N¹, p. 147-155.

SKAKUN, S., KUSSUL, N., SHELESTOV, A. Y., LAVRENIUK, M., \& KUSSUL, O. Efficiency assessment of multitemporal C-band Radarsat-2 intensity and Landsat-8 surface reflectance satellite imagery for crop classification in Ukraine. IEEE Journal of Selected Topics in Applied Earth Observations and Remote Sensing. 2016. Vol. 9, № 8, p. 3712-3719.

STEHMAN, S. V. Selecting and interpreting measures of thematic classification accuracy. Remote sensing of Environment. 1997. Vol. 62, N 1, p. 77-89.

SUÁREZ, J. E., DELGADO, C., ALBERICH, M. A., \& CALDERÓN, G. Curvas de daños económicos provocados por inundaciones en zonas habitacionales y agrícolas de México. Parte II: caso de estudio en la cuenca alta del río Lerma, México. Tecnología y ciencias del agua. 2007. Vol. 22, $\mathrm{N}^{\circ} 3$, p. $71-85$.

TAPIA-SILVA, F., ITZEROTT, S., FOERSTER, S., KUHLMANN, B., \& KREIBICH, H. Estimation of flood losses to agricultural crops using remote sensing. Physics and Chemistry of the Earth, Parts $A / B / C$. January 2011. Vol. 36, N 7-8, p. 253-265. http://DOI 10.1016/j.pce.2011.03.005.

TEFERI, E., UHLENBROOK, S., BEWKET, W., WENNINGER, J., \& SIMANE, B. The use of remote sensing to quantify wetland loss in the Choke Mountain range, Upper Blue Nile basin, Ethiopia. Hydrology and Earth System Sciences. 3 December 2010. Vol. 14, № 12, p. 2415-2428.

TERRSET VERSIÓN 1831. Clark Labs. 2018.

TWELE, A., CAO, W., PLANK, S., \& MARTINIS, S. Sentinel-1-based flood mapping: a fully automated processing chain. International Journal of Remote Sensing. 2016. Vol. 37, N 13, p. 2990-3004.

VAN DER SANDE, C.J., DE JONG, S. M., \& DE ROO, A. P. J. A segmentation and classification approach of IKONOS-2 imagery for land cover mapping to assist flood risk and flood damage assessment. International Journal of applied earth observation and geoinformation. 2003. Vol. 4, $N^{\circ} 3$, p. 217-229.

VOZINAKI, A. K., KARATZAS, G. P., SIBETHEROS, I. A., \& VAROUCHAKIS, E. A. An agricultural flash flood loss estimation methodology: the case study of the Koiliaris basin (Greece), February 2003 flood. Natural Hazards. November 2015. Vol. 79, N² 2, p. 899-920.

WORLD BANK. World Development Indicators. 2020. (Consulta 11/01/20). Available from: https:// datos.bancomundial.org/ 
$\mathrm{XU}, \mathrm{H}$. Modification of normalised difference water index (NDWI) to enhance open water features in remotely sensed imagery. International Journal of Remote Sensing, 2006. Vol. 27, No 14 , p. 3025-3033.

ZANTER, K. Landsat 8 (L8) data users handbook. Landsat Science Official Website. 2016.

ZHANG, N., HONG, Y., QIN, Q., \& LIU, L. VSDI: a visible and shortwave infrared drought index for monitoring soil and vegetation moisture based on optical remote sensing. International Journal of Remote Sensing, 2013. Vol. 34, N 13, p. 4585-4609.

ZHOU, S.L., \& ZHANG, W.C. Flood monitoring and damage assessment in Thailand using multi-temporal HJ-1A/1B and MODIS images. In : IOP Conference Series: Earth and Environmental Science. IOP Publishing, 2017. p. 012016. 
